\title{
Mass Compression from Recurrent Lymphoma Mimicking Lower Extremity Cellulitis
}

\author{
David G. Li ${ }^{1}$, Katherine M. Krajewski ${ }^{2}$, Arash Mostaghimi ${ }^{1}$ \\ 1. Department of Dermatology, Brigham \& Women's Hospital, Harvard Medical School, Boston, USA 2. Department of \\ Imaging, Dana-Farber Cancer Institute; Department of Radiology, Brigham and Women's Hospital, Harvard Medical \\ School, Boston, USA
}

Corresponding author: David G. Li, dgli@bwh.harvard.edu

\begin{abstract}
Cellulitis is a common skin and soft tissue infection with substantial misdiagnosis rates due to its nonspecific clinical characteristics. In this report, we present a patient with recurrent metastatic diffuse large B-cell lymphoma (DLBCL) masquerading as a unilateral lower extremity cellulitis. A 62-year-old man with a history of DLBCL, in remission for two years, presented with a two-week history of swelling and erythema of the right thigh and leg. Despite treatment with clindamycin and cephalexin, the redness and swelling continued to progress. On presentation to the emergency department, vitals were within normal limits, laboratory workup was significant only for borderline anemia and thrombocytopenia, and bilateral lower extremity ultrasound was negative for a clot. The patient was evaluated by a dermatologist who recommended further imaging workup for proximal vascular compression given the uniformity of inflammation and edema in the absence of fever or systemic symptoms. Imaging revealed retroperitoneal lymphadenopathy, right pelvic side wall and right inguinal lymphadenopathy, an intramuscular lymphomatous involvement of the right iliopsoas muscle, and mass compression of the right external iliac vein. Bone marrow and soft-tissue biopsies confirmed recurrent and metastatic DLBCL. In this patient, the atypical cellulitis-like features are likely due to venous and lymphatic obstruction secondary to mass effect from metastasis. Going forward, clinicians should consider compression-induced edema as a sign of primary or recurrent malignancy in patients with refractory or atypical cellulitis.
\end{abstract}

Categories: Dermatology, Oncology

Keywords: cellulitis, pseudocellulitis, lymphoma, compression, diffuse large b-cell lymphoma (dlbcl), cancer, erythema, edema

Received 03/05/2018 Review began 03/07/2018 Review ended 04/09/2018 Published 04/12/2018

(c) Copyright 2018 Li et al. This is an open access article distributed under the terms of the Creative Commons Attribution License CC-BY 3.0., which permits unrestricted use, distribution, and reproduction in any medium, provided the original author and source are credited.

\section{Introduction}

Cellulitis is an infection of the deep dermis and subcutaneous tissue that often presents as expanding erythema, swelling, warmth, and tenderness of the affected site [1]. The lack of a "gold standard" diagnostic modality and nonspecific clinical characteristics results in frequent misdiagnosis of cellulitis mimickers (pseudocellulitis) as cellulitis, leading to inappropriate antibiotic therapy and potential complications [2]. In this report, we present a case of recurrent metastatic diffuse large B-cell lymphoma (DLBCL) mimicking a unilateral lower extremity cellulitis.

\section{Case Presentation}

A 62-year-old man with a history of DLBCL previously treated with autologous stem cell transplant and combination chemotherapy with rituximab, in remission for two years, presented with a two-week history of swelling and erythema of the right thigh and leg. The symptoms began acutely as an erythematous patch on his right thigh, at which time he was started on oral clindamycin for presumed cellulitis. Despite a sevenday-course of clindamycin, the patient reported progression of erythema down his leg and was subsequently transitioned to three days of oral cephalexin, also without improvement.

On presentation, the patient had confluent erythema and mild edema from the right upper thigh to the medial calf without tenderness or pruritus (Figures 1-2). His blood pressure was $123 / 62 \mathrm{mmHg}$; heart rate 83/minute; temperature $37.0 \mathrm{C}$ (98.6 F); respiratory rate 18; and oxygen saturation 97\%. Complete blood count was remarkable only for borderline anemia and thrombocytopenia. The patient's asymmetry, leukocytosis, tachycardia, age $\geqslant \underline{70}$ (ALT-70) lower extremity cellulitis score was three (unilateral disease, no leukocytosis or tachycardia, age $<70$ ), an indeterminate finding that may benefit from further workup or consultation [3]. Ultrasound evaluation of the lower extremities for clot was unremarkable. The patient was evaluated by dermatology during this encounter, who recommended imaging workup for possible proximate vascular compression considering the uniformity of inflammation and edema in absence of fever or systemic symptoms. 


\section{Cureus}

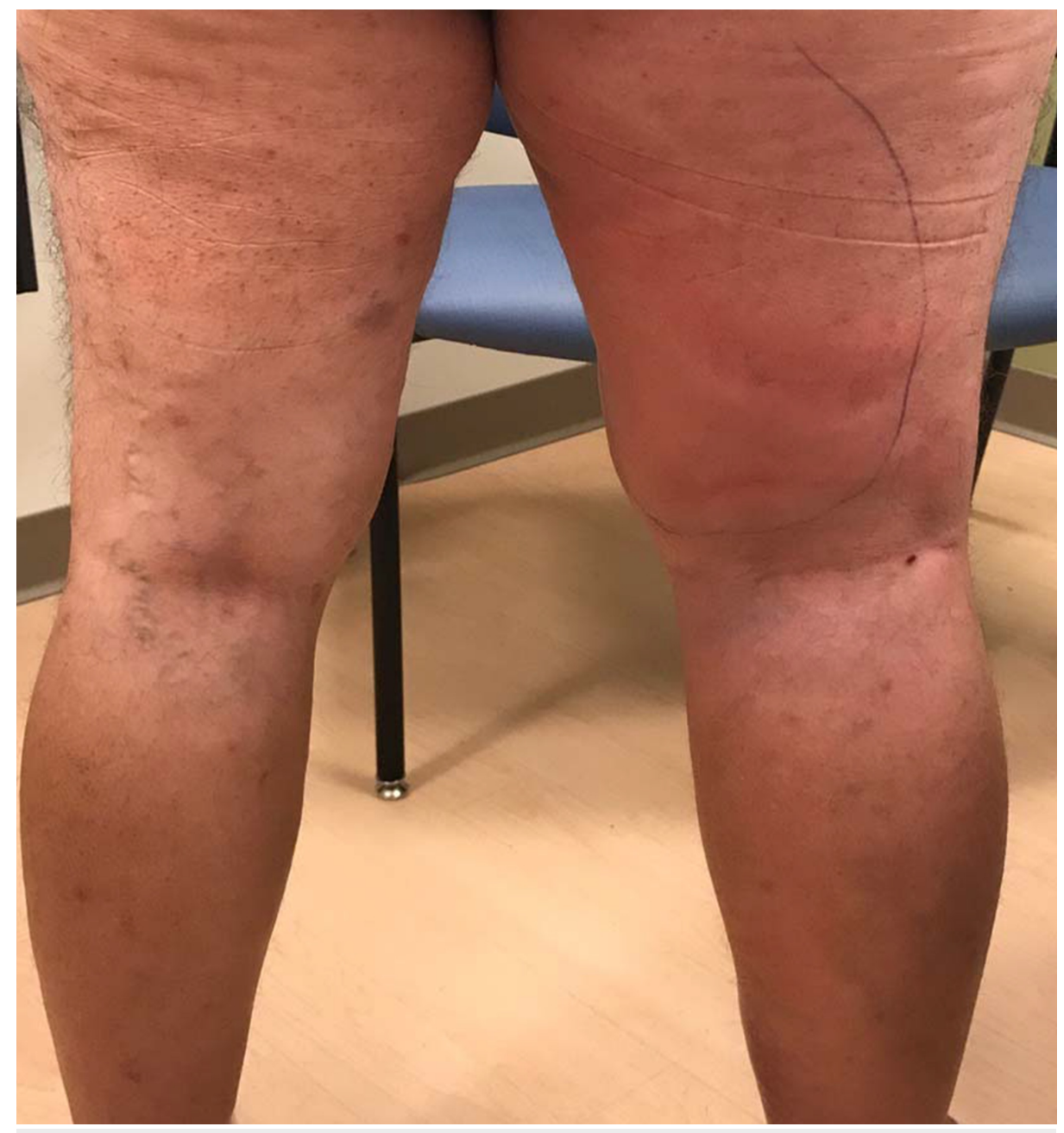

FIGURE 1: Compression lymphadenopathy mimicking cellulitis One confluent and edematous plaque over the right posterior thigh. 


\section{Cureus}

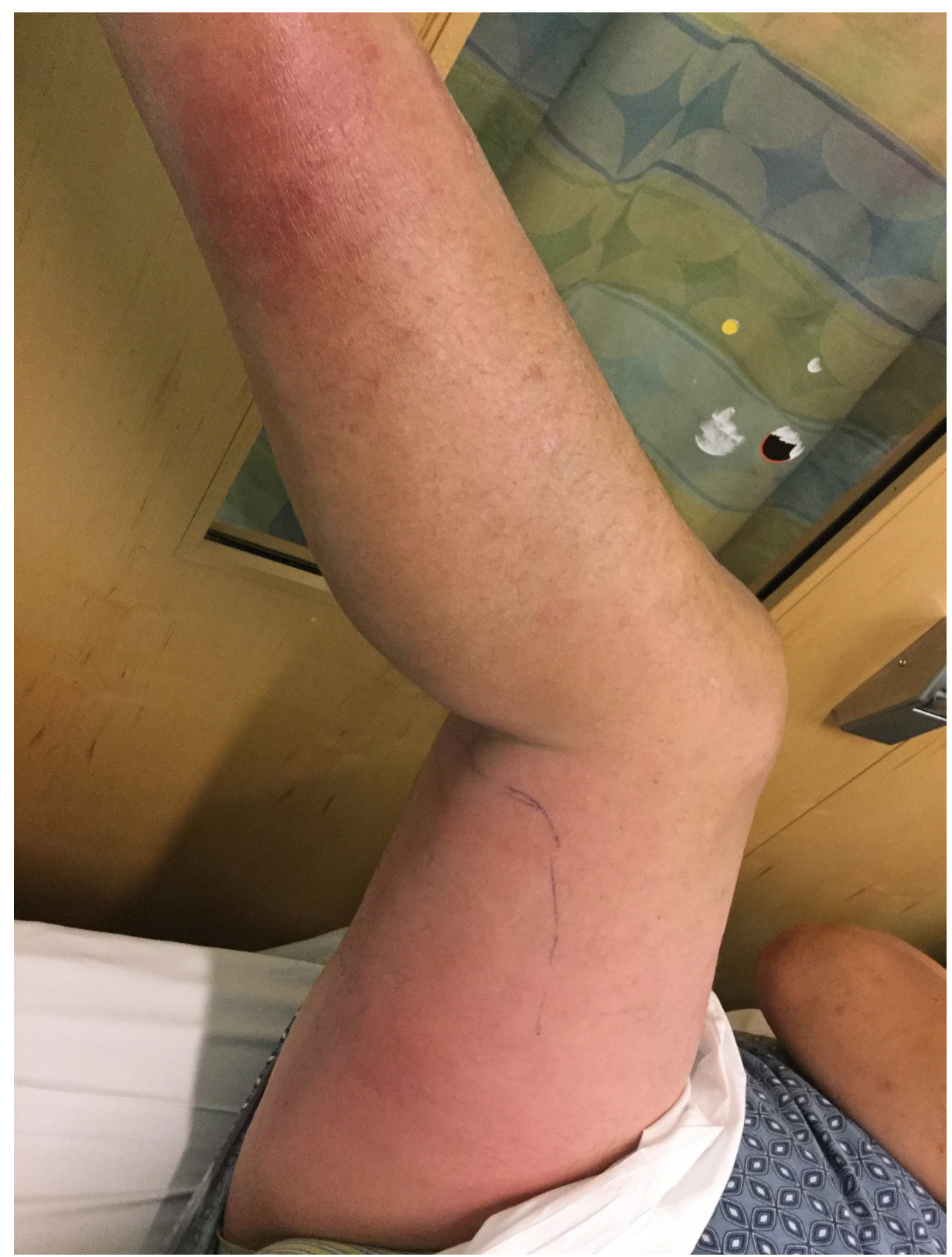

\section{FIGURE 2: Compression lymphadenopathy mimicking cellulitis}

Poorly demarcated erythematous and edematous plaques over the right posteromedial leg and right posterior thigh extending to the right buttocks.

Computed tomography (CT) of the abdomen and pelvis was performed and was remarkable for retroperitoneal lymphadenopathy (Figure 3). Magnetic resonance imaging (MRI) showed lymphadenopathy of the right pelvic side wall and right inguinal regions, intramuscular lymphomatous involvement of the right iliopsoas muscle, and mass compression of the right external iliac vein (Figure 4). Antibiotics were discontinued and the patient improved with leg elevation. Bone marrow and soft-tissue biopsies (skin biopsy not performed) after discharge confirmed recurrent and metastatic DLBCL. 


\section{Cureus}

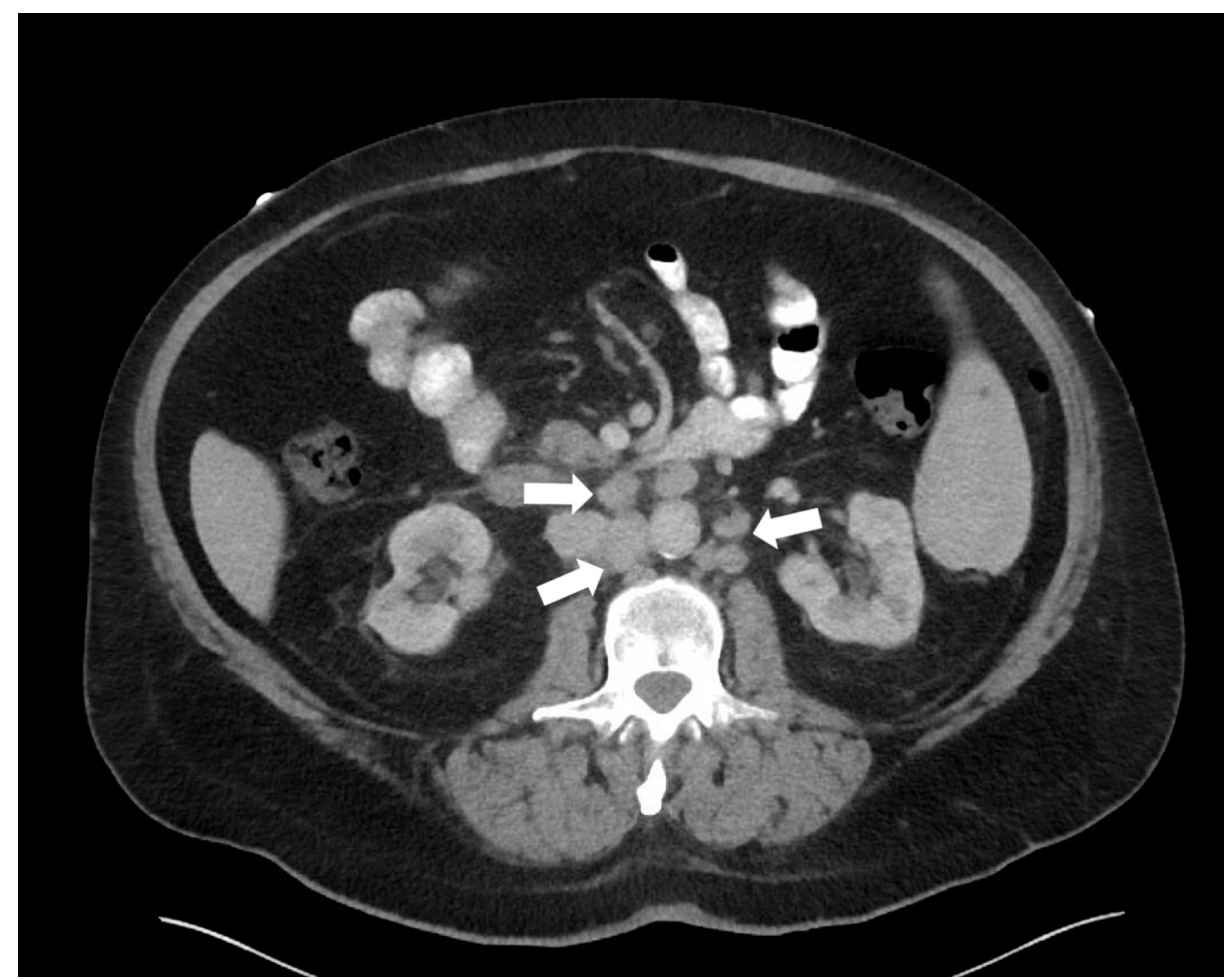

FIGURE 3: Recurrent diffuse large B-cell lymphoma

Computed tomography (CT) demonstrating retroperitoneal adenopathy (arrows). 


\section{Cureus}

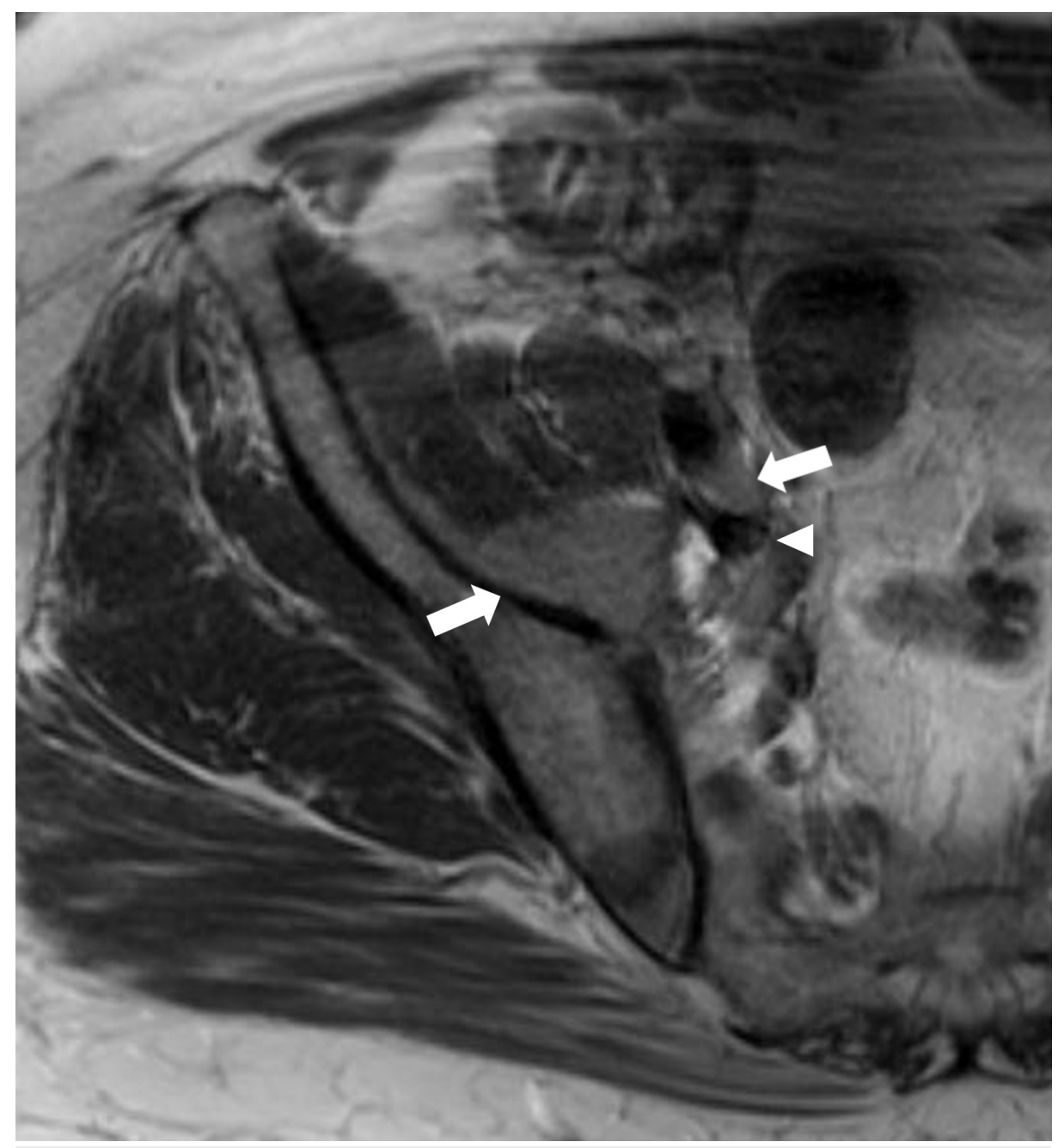

\section{FIGURE 4: Recurrent diffuse large B-cell lymphoma}

Magnetic resonance imaging (MRI) revealing hyperintense lymphoma lesions involving the right iliopsoas muscle (left sided arrow), right external iliac node (right sided arrow) with subsequent compression of the right external iliac vein (arrowhead).

\section{Discussion}

A subset of diseases resembling cellulitis (aka pseudocellulitis) has been previously documented in the context of malignancy, including carcinoma erysipeloides (cutaneous metastasis of visceral carcinomas such as pulmonary or prostate adenocarcinoma) presenting as erythematous, infiltrative patches, and plaques with local inflammation [4-5]. Cellulitis-like Sweets Syndrome, a neutrophilic dermatosis, has been documented to precede the discovery of a malignant neoplasm including myeloproliferative disease [6]. Bone metastasis in patients with underlying malignancy have also manifested as pseudocellulitis, leading to delays in care and unnecessary antimicrobial therapy [7].

Acute compression secondary to malignancy, as described in the present case, should also be added to the differential for pseudocellulitis. Increased awareness of cutaneous pseudocellulitis as a manifestation of underlying malignancy, especially in high-risk patients (e.g., patients at risk for recurrence, with family history of cancer, or past exposure to carcinogens) [8], is important not only to avoid unnecessary antibiotics and hospitalization, but also to begin appropriate and timely therapy for malignancy.

While there have also been reports of DLBCL mimicking cellulitis [9-10], the present case differs in the mechanism responsible for pseudocellulitis features. In our patient, the atypical cellulitis-like features are likely the result of venous and lymphatic obstruction (involving the right external iliac vein and inguinal/pelvic side wall lymph nodes, respectively) secondary to mass effect from metastasis. Although the patient presented with features suggestive of cellulitis, there was a progression of symptoms despite a tenday course of two different oral antibiotics. Although the lack of response to multiple antibiotics decreases the likelihood of cellulitis, it does not eliminate the possibility of resistant or atypical microorganisms. However, the patient denied fevers or chills, was hemodynamically stable, had no leukocytosis, and had an 
ALT-70 score of three, which was indeterminate and would likely benefit from specialty consultation [3].

\section{Conclusions}

In this patient who did not respond to a standard cellulitis treatment protocol, the atypical cellulitis-like features are likely due to venous and lymphatic obstruction secondary to mass effect from metastasis, as seen from clinical and radiological evidence. Going forward, clinicians should consider compression-induced edema as a sign of primary or recurrent malignancy in patients with refractory or atypical cellulitis.

\section{Additional Information \\ Disclosures}

Human subjects: Consent was obtained by all participants in this study. Partners Healthcare IRB issued approval n/a. Not applicable. . Conflicts of interest: In compliance with the ICMJE uniform disclosure form, all authors declare the following: Payment/services info: All authors have declared that no financial support was received from any organization for the submitted work. Financial relationships: All authors have declared that they have no financial relationships at present or within the previous three years with any organizations that might have an interest in the submitted work. Other relationships: All authors have declared that there are no other relationships or activities that could appear to have influenced the submitted work.

\section{Acknowledgements}

This study was supported by the TL1 Award (DGL) sponsored by the National Center for Advancing Translational Sciences, National Institutes of Health, Award Number TL1TR001062.

\section{References}

1. Raff AB, Kroshinsky D: Cellulitis: a review. JAMA. 2016, 316:325-337. 10.1001/jama.2016.8825

2. Weng QY, Raff AB, Cohen JM, et al.: Costs and consequences associated with misdiagnosed lower extremity cellulitis. JAMA Dermatol. 2017, 153:141-146. 10.1001/jamadermatol.2016.3816

3. Raff AB, Weng QY, Cohen JM, et al.: A predictive model for diagnosis of lower extremity cellulitis: a crosssectional study. J Am Acad Dermatol. 2017, 76:618-625. 10.1016/j.jaad.2016.12.044

4. Yu WW, Jang CS: Carcinoma erysipeloides, a rare presentation of cutaneous metastasis from pulmonary adenocarcinoma. Dermatologica Sinica. 2017, 35:159-160. 10.1016/j.dsi.2017.02.001

5. Ng CS: Carcinoma erysipeloides from prostate cancer presenting as cellulitis . Cutis. 2000, 65:215-216.

6. Surovy AM, Pelivani N, Hegyi I, et al.: Giant cellulitis-like sweet syndrome, a new variant of neutrophilic dermatosis. JAMA Dermatol. 2013, 149:79-83. 10.1001/2013.jamadermatol.548

7. Kashima J, Okuma Y, Watanabe K, et al.: Cellulitis-like symptoms manifested by bone metastasis of lung cancer in a patient living with human immunodeficiency virus. Int J STD AIDS. 2016, 27:912-916. $10.1177 / 0956462415606014$

8. Risk Factors for Cancer. (2015). Accessed: March 2, 2018: https://www.cancer.gov/about-cancer/causesprevention/risk.

9. Pan ST, Wei CH, Kuo HY, et al.: Primary cutaneous diffuse large b-cell lymphoma, leg type mimicking cellulitis. Dermatologica Sinica. 2013, 31:104-106. 10.1016/j.dsi.2012.10.006

10. Scott JF, Honda K, Lu K: Seemed like cellulitis: intravascular large b-cell lymphoma. Am J Med. 2016, 129:685-687. 10.1016/j.amjmed.2016.03.001 